\title{
A new mathematical model for environmental monitoring and assessment
}

\author{
Roberto Monaco \\ DIST, Politecnico di Torino, Viale Mattioli 39, 10125 Torino, Italy \\ Ana Jacinta Soares \\ CMAT, University of Minho, Campus de Gualtar, 4710-057 Braga, Portugal
}

(February 3, accepted in PSPDES IV, 2015)

\begin{abstract}
In this paper we are concerned with a quantitative method of Landscape Ecology. More in details we consider an environmental system distributed in landscape units (ecological sectors) and we propose a new mathematical model in order to implement a method for the evaluation of the ecological state of the system under investigation. After having performed a stability analysis of the model, we apply the proposed procedure first by considering separately each landscape unit and then extending our investigation to the system as a whole, by taking into account the connections between all the landscape units themselves. Our investigation includes some numerical computations that were performed for a Northern district of the Turin Province, using an approximation procedure that should avoid stiffness problems.
\end{abstract}

Keywords. Landscape ecology. Mathematical modeling. Environmental dynamics. Stability analysis. Numerical computations.

\section{Introduction}

For a quantitative evaluation of the ecological state of an environmental system an important contribution is given in the book [11]. Other important contributions to this matter can be found as well in the paper [19] and in the book [5] where for the first time the use of the so-called ecological graph is proposed. For a quantitative description of a territory relevant indicators have been also proposed in the references $[16,15]$ where ideas coming from the mathematical theory of communication [17] and from conceptual models [1] have been transposed to Landscape Ecology.

In the quoted bibliography, as we shall see, the environment is distributed in different landscape units (often mentioned in what follows as ecological sectors) which can be more or less connected by flows of material and biological energy. In this sense a relevant variable to describe the territory is certainly the last quantity which can be coupled to the consistency of green areas of high ecological quality.

In the past years some mathematical models useful for a quantitative monitoring of environments have been proposed in a couple of papers $[8,9]$. In particular in the former a mathematical model, with only two state equations that consider the environment as a whole, has been deduced, assuming as state variables the bio-energy and the percentage of green areas in the territory under investigation.

In the latter such a model has been studied in terms of its stability analysis, finding also bifurcations and discussing on its qualitative properties. Moreover in paper [9], as a future perspective, a new model including state variables at the level of landscape units has been suggested. These models, on the mathematical ground, are represented by an autonomous system of evolution ordinary differential equations, whose equilibrium solutions express the future scenarios of the environment itself.

Starting from this last idea in the present paper we present a model where each landscape unit is represented by two time-dependent variables, namely the extent of green areas of high ecological quality 
and a suitable function depending on the biological energy per year. Such a new version of the model is capable to identify the characters of the territory at a more detailed level, so that the territory itself results to be more readable.

For a first moment, in Section 2, we are concerned with the model stability analysis, considering separately the landscape units. We then apply, in Section 3, such an analysis to a Northern district of the Turin Province. This district has been previously studied by De Palma in her master degree thesis [4], deriving all the relevant indicators of this environmental system. Moreover, in Sections 4 and 5 , we complete the environment analysis by coupling all together the landscape units, showing that, in the present case study, connectivity plays a crucial role. For this last analysis the model becomes rather cumbersome and may present some instabilities since it includes 48 coupled ordinary differential equations. For this reason in Section 4 we propose an explicit approximation procedure, discussed in paper [10] starting from the methods presented in the book [12], and bibliography therein cited. Finally, conclusions and some ideas for future work are presented in Section 6.

\section{The mathematical modeling for landscape units: equilibrium solutions and stability}

An environmental system is an isolated system that may be distributed in $n$ landscape units (LU) divided by natural or anthropological barriers (roads, motorways, railways, buildings, industrial infrastructures, rivers, hill ridges and so on). At the same time a LU is formed by several biotopes which are patches characterized by an uniform land cover. Such a representation of an environment can be given by the Geographic Information System (GIS) [7] and its ecological indicators [3] can be deduced by the ecological graph (see its construction in the book [5] and in the paper [8]).

The nature of each biotope is identified by the value of its bio-potential territorial capacity (BTC) index [11]. In what follows, such an index, measuring the biological energy per year and per square meter, produced by the vegetation inside the biotope, will be indicated by $B_{j i}, j=1, \ldots, q_{i}$, being $i$ a subscript which indicates that the $j$-th biotope belongs to the $i$-th $\mathrm{LU}, i=1, \ldots, n$. The variable $B_{j i}$ assumes values $[8,11]$ in the range $\left[0, B_{\max }\right]$ where $B_{\max }=6.5 \mathrm{Mcal} /\left(\mathrm{m}^{2} \cdot\right.$ year $)$ at the European latitudes and corresponds to oak woods.

Moreover the BTC index identifies five ecological classes distributed as follows

$$
\mathcal{C}_{1}=[0,0.4), \mathcal{C}_{2}=[0.4,1.2], \mathcal{C}_{3}=(1.2,2.4], \mathcal{C}_{4}=(2.4,4.0], \mathcal{C}_{5}=(4.0,6.5]
$$

The total value of BTC of each LU, in Mcal/year, is given by

$$
B_{i}(t)=\sum_{j=1}^{q_{i}} B_{j i} s_{j i}
$$

where $s_{j i}$ is the area of the biotope $j$. Accordingly the area of the corresponding LU is $S_{i}=\sum_{j=1}^{q_{i}} s_{j i}$.

To the BTC is often associated another quantity that can be interpreted as a generalized function of BTC (GBTC) defined by the following formula [5]

$$
M_{i}(t)=\left(1+K_{i}\right) B_{i}(t)
$$

where the constant parameters $K_{i} \in[0,1]$ depend upon the physical and morphological features of the LU in such a way that the BTC itself is incremented. Such an increment takes into account the capacity of the LU to transmit energy to the neighboring LUs. In this paper $K_{i}$ will be expressed in terms of three other parameters

$$
K_{i}=\left(K_{i}^{s h}+K_{i}^{e c}+K_{i}^{p e}\right) / 3,
$$

where $K_{i}^{s h}, K_{i}^{e c}, K_{i}^{p e} \in[0,1]$ are, respectively, the Shannon landscape diversity parameter (evenness $[16,15])$, the ecotonal parameter [3] and the LU-border permeability parameter [8]. They are defined as follows: 
- the Shannon parameter

$$
K_{i}^{s h}=\left(\sum_{\ell=1}^{5} \frac{n_{\ell i}}{5} \log \frac{n_{\ell i}}{5}\right) / \log \frac{1}{5},
$$

where $n_{\ell i}$ is the number of biotopes belonging to the ecological class $\mathcal{C}_{\ell}, \ell=1, \ldots, 5$, assuming that $n_{\ell i} \log n_{\ell i}=0$ if $n_{\ell i}=0$;

- the ecotonal parameter (i.e. the length of borders between biotopes)

$$
K_{i}^{e c}=1-P_{i} / \sum_{j=1}^{q_{i}} P_{j i},
$$

where $P_{i}$ is the perimeter of the $i$-th $\mathrm{LU}$ and $P_{j i}$ are the perimeters of all the biotopes except those belonging to the ecological class $\mathcal{C}_{1}$;

- the LU-border permeability parameter

$$
K_{i}^{p e}=\frac{1}{P_{i}} \sum_{r=1}^{s} L_{i}^{r} p^{r},
$$

where $L_{i}^{r}$ is the length of the portion $r$ of the border, divided in $s$ parts, and $p^{r} \in[0,1]$ is the permeability index, with $p=0$ for an impermeable barrier, and $p=1$ for a complete permeable barrier (see $[8,11])$.

According to previous versions of the mathematical model already indicated in the Introduction $[8,9]$, the state variables for each $\mathrm{LU}$ of the present model are two, precisely the area $V_{i}(t)$ of the biotopes belonging to the ecological classes $\mathcal{C}_{4}$ and $\mathcal{C}_{5}$ (high ecological quality of green) and the GBTC $M_{i}(t)$. The right-hand-side of the evolution equations on $V_{i}(t)$ and $M_{i}(t)$ consists in a gain term of logistic type and a loss term accounting for environmental impact. The equations have the following form

$$
\begin{gathered}
V_{i}^{\prime}(t)=b_{i}\left(M_{i}\right)\left(1-\frac{V_{i}(t)}{S_{i}}\right) V_{i}(t)-h_{i} U_{i} V_{i}(t), \\
M_{i}^{\prime}(t)=c_{i}\left(1-\frac{M_{i}(t)}{M_{i}^{\text {max }}}\right) M_{i}(t)-r_{i}\left(1-\frac{V_{i}(t)}{S_{i}}\right) M_{i}(t) .
\end{gathered}
$$

In equation (3) the coefficient $b_{i}$ will be expressed, as we shall see, in terms of the GBTC, whereas the other parameters $h_{i}$ and $U_{i}$ are, respectively, given by the ratio between the sum of the perimeters of the built-up areas and the total perimeter of the LU, and by the ratio between the sum of the built-up areas and the total area $S_{i}$ of the LU. Therefore, these parameters, assumed as constant, can be considered a measure, respectively, of the dispersion and of the intensity of edification inside the LU. According to its definition the parameter $h_{i}$ can assume values greater than one (values greater than one mean that edification dispersion in the LU is significantly remarkable); on the other hand $U_{i}$ is ranging in $[0,1]$. In equation (4) the coefficient $c_{i}$ is the connectivity index between the $i$-th $\mathrm{LU}$ and its neighbors, whereas the other parameter $r_{i}$ is defined as the ratio between the surface area of the impermeable barriers present in the LU and the total area $S_{i}$ of the LU itself. Both parameters are supposed to range in $[0,1]$ and for this preliminary analysis the indexes $c_{i}$ are assumed to be constant. Let us now deal with the definition of the parameter $b_{i}\left(M_{i}\right)$, i.e.

$$
b_{i}:=\frac{B_{i}}{B_{i}^{\max }}, \quad B_{i}^{\max }=B_{\max } S_{i} .
$$

Thus $b_{i}$ expresses the production percentage of BTC with respect to the maximum value that each LU can produce, assuming that all its biotopes have BTC index equal to $B_{\max }$. If now we define the normalized GBTC

then we have

$$
m_{i}:=\frac{M_{i}}{M_{i}^{\max }}, \quad M_{i}^{\max }=2 B_{i}^{\max },
$$

$$
m_{i}=\frac{\left(1+K_{i}\right) B_{i}}{2 B_{i}^{\text {max }}}=\frac{1+K_{i}}{2} b_{i}
$$


and therefore

$$
b_{i}=\frac{2}{1+K_{i}} m_{i}:=a_{i} m_{i}, \quad a_{i} \in[1,2] .
$$

If now we normalize $V_{i}$ as well, defining $v_{i}:=V_{i} / S_{i}$, both state variables $v_{i}$ and $m_{i}$ range in $[0,1]$. Thus if we divide Eqs. (3-4), respectively, by $S_{i}$ and $M_{i}^{\max }$, the model equations assume the following final form

$$
\begin{gathered}
v_{i}^{\prime}(t)=a_{i} m_{i}(t)\left[1-v_{i}(t)\right] v_{i}(t)-h_{i} U_{i} v_{i}(t), \\
m_{i}^{\prime}(t)=c_{i}\left[1-m_{i}(t)\right] m_{i}(t)-r_{i}\left[1-v_{i}(t)\right] m_{i}(t) .
\end{gathered}
$$

Moreover to these equations we join the initial data

$$
v_{i}(t=0)=v_{i 0}, \quad m_{i}(t=0)=m_{i 0},
$$

which must be determined directly from the GIS maps of the environment under investigation.

Next step consists in finding the equilibrium solutions [13] of the system (6-7). Solving the algebraic equations

$$
v_{i}\left[a_{i} m_{i}\left(1-v_{i}\right)-h_{i} U_{i}\right]=0, \quad m_{i}\left[c_{i}\left(1-m_{i}\right)-r_{i}\left(1-v_{i}\right)\right]=0,
$$

one obtains the following equilibria.

- The first is given by

$$
\left(v_{i}^{(1)}=0, m_{i}^{(1)}=0\right),
$$

which corresponds to a scenario where the environment tends to lose substantially its ecological quality since it is characterized by a strong fragmentation;

- the second is expressed by

$$
\left(v_{i}^{(2)}=0, m_{i}^{(2)}=\frac{c_{i}-r_{i}}{c_{i}}\right),
$$

corresponding to a scenario of weak ecological quality characterized by a moderate level of bio-energy (such a scenario is typical of a territory with a predominant agricultural production); the admissibility condition of such solution results to be $c_{i}>r_{i}$;

- the third and fourth equilibria are those of coexistence, showing a good level of bio-energy production together with a high ecological quality of green areas; they are given by

$$
\begin{aligned}
& \left(v_{i}^{(3)}=\frac{2 r_{i}-c_{i}-D_{i}}{2 r_{i}}, m_{i}^{(3)}=\frac{2 h_{i} U_{i} r_{i}}{a_{i}\left(c_{i}+D_{i}\right)}\right) \\
& \left(v_{i}^{(4)}=\frac{2 r_{i}-c_{i}+D_{i}}{2 r_{i}}, m_{i}^{(4)}=\frac{2 h_{i} U_{i} r_{i}}{a_{i}\left(c_{i}-D_{i}\right)}\right)
\end{aligned}
$$

where

$$
D_{i}=\sqrt{c_{i}\left(a_{i} c_{i}-4 h_{i} U_{i} r_{i}\right) / a_{i}} .
$$

The existence of these solutions requires that

$$
c_{i}>\frac{4 h_{i} U_{i} r_{i}}{a_{i}} .
$$

Moreover the third solution exists if

$$
\frac{1}{2}\left(c_{i}+D_{i}\right)<r_{i}<\frac{1}{2}\left(c_{i}+D_{i}\right) \frac{a_{i}}{h_{i} U_{i}},
$$

whereas the fourth requires that

$$
c_{i}>D_{i}, \quad \frac{1}{2}\left(c_{i}-D_{i}\right)<r_{i}<\frac{1}{2}\left(c_{i}-D_{i}\right) \frac{a_{i}}{h_{i} U_{i}} .
$$


We are now concerned with asymptotic stability [13] of the previous equilibria. The Jacobian matrix joined to the system (6-7) is given by

$$
J\left(v_{i}, m_{i}\right)=\left(\begin{array}{cc}
a_{i} m_{i}-2 a_{i} v_{i} m_{i}-h_{i} U_{i} & a_{i}\left(1-v_{i}\right) v_{i} \\
r_{i} m_{i} & c_{i}-2 c_{i} m_{i}-r_{i}\left(1-v_{i}\right)
\end{array}\right) .
$$

1) We have for the first equilibrium

$$
J\left(v_{i}^{(1)}, m_{i}^{(1)}\right)=\left(\begin{array}{cc}
-h_{i} U_{i} & 0 \\
0 & c_{i}-r_{i}
\end{array}\right) .
$$

The eigenvalues of the matrix are $\lambda_{1}=-h_{i} U_{i}<0$ and $\lambda_{2}=c_{i}-r_{i}$. Thus the equilibrium solution $\left(v_{i}^{(1)}, m_{i}^{(1)}\right)$ is asymptotically stable if $c_{i}<r_{i}$; otherwise it is a saddle point. Moreover the stability of this solution implies that the equilibrium $\left(v_{i}^{(2)}, m_{i}^{(2)}\right.$ ) does not exist (see the admissibility condition of the second equilibrium).

2) For the second equilibrium we get

$$
J\left(v_{i}^{(2)}, m_{i}^{(2)}\right)=\left(\begin{array}{cc}
\frac{a_{i}\left(c_{i}-r_{i}\right)-h_{i} U_{i} c_{i}}{c_{i}} & 0 \\
\frac{r_{i}\left(c_{i}-r_{i}\right)}{c_{i}} & -c_{i}+r_{i}
\end{array}\right),
$$

whose eigenvalues are $\lambda_{1}=\frac{a_{i}\left(c_{i}-r_{i}\right)-h_{i} U_{i} c_{i}}{c_{i}}$ and $\lambda_{2}=-c_{i}+r_{i}$. Existence of this equilibrium implies that the eigenvalue $\lambda_{2}$ is always negative. Thus the asymptotic stability of $\left(v_{i}^{(2)}, m_{i}^{(2)}\right)$ requires that $a_{i} c_{i}<a_{i} r_{i}+h_{i} U_{i} c_{i}$.

3) Moreover for the third equilibrium we have

$$
J\left(v_{i}^{(3)}, m_{i}^{(3)}\right)=\left(\begin{array}{cc}
\frac{h_{i} U_{i}\left(c_{i}+D_{i}-2 r_{i}\right)}{c_{i}+D_{i}} & \frac{a_{i}\left(c_{i}+D_{i}\right)\left(2 r_{i}-c_{i}-D_{i}\right)}{4 r_{i}^{2}} \\
\frac{2 h_{i} U_{i} r_{i}^{2}}{a_{i}\left(c_{i}+D_{i}\right)} & \frac{a_{i}\left(c_{i}^{2}-D_{i}^{2}\right)-8 h_{i} U_{i} c_{i} r_{i}}{2 a_{i}\left(c_{i}+D_{i}\right)}
\end{array}\right) .
$$

If we write the characteristic equation of the Jacobian in the form $\lambda^{2}+A_{1} \lambda+B_{1}=0$, after simple computations we get

$$
\begin{gathered}
A_{1}=-\frac{2 a_{i} h_{i} U_{i}\left(c_{i}+D_{i}-2 r_{i}\right)+a_{i}\left(c_{i}^{2}-D_{i}^{2}\right)-8 h_{i} U_{i} c_{i} r_{i}}{2 a_{i}\left(c_{i}+D_{i}\right)} \\
B_{1}=\frac{h_{i} U_{i}\left(c_{i}+D_{i}-2 r_{i}\right)\left[a_{i} c_{i}\left(c_{i}+D_{i}\right)-4 h_{i} U_{i} c_{i} r_{i}\right]}{a_{i}\left(c_{i}+D_{i}\right)^{2}} .
\end{gathered}
$$

Therefore the stability condition for the third equilibrium requires that $A_{1}>0$ and $B_{1}>0$. Conversely, if $A_{1}>0$ and $B_{1}<0$ or if $A_{1}<0$ and $B_{1}<0$, we have that such an equilibrium is a saddle point, whereas if $A_{1}<0$ and $B_{1}>0$ we have instability. In addition, in the case of stability, if $A_{1}^{2}-4 B_{1}>0$ the equilibrium is a node, whereas $A_{1}^{2}-4 B_{1}<0$ corresponds to a focus. In cases that $A_{1}=0$ or $B_{1}=0$, the asymptotic stability is not assured. Nevertheless, in practice, since $A_{1}$ and $B_{1}$ depend on territorial indexes of different nature, the vanishing of these quantities is strongly unlikely and not consistent with the real state of the environment.

4) Finally for the last equilibrium $\left(v_{i}^{(4)}, m_{i}^{(4)}\right)$ the Jacobian has the form

$$
J\left(v_{i}^{(4)}, m_{i}^{(4)}\right)=\left(\begin{array}{cc}
\frac{h_{i} U_{i}\left(c_{i}-D_{i}-2 r_{i}\right)}{c_{i}-D_{i}} & \frac{a_{i}\left(c_{i}-D_{i}\right)\left(2 r_{i}-c_{i}+D_{i}\right)}{4 r_{i}^{2}} \\
\frac{2 h_{i} U_{i} r_{i}^{2}}{a_{i}\left(c_{i}-D_{i}\right)} & \frac{a_{i}\left(c_{i}^{2}-D_{i}^{2}\right)-8 h_{i} U_{i} c_{i} r_{i}}{2 a_{i}\left(c_{i}-D_{i}\right)}
\end{array}\right),
$$


and the coefficients of its characteristic equation $\lambda^{2}+A_{2} \lambda+B_{2}=0$ are given by

$$
\begin{gathered}
A_{2}=-\frac{2 a_{i} h_{i} U_{i}\left(c_{i}-D_{i}-2 r_{i}\right)+a_{i}\left(c_{i}^{2}-D_{i}^{2}\right)-8 h_{i} U_{i} c_{i} r_{i}}{2 a_{i}\left(c_{i}-D_{i}\right)} \\
B_{2}=\frac{h_{i} U_{i}\left(c_{i}-D_{i}-2 r_{i}\right)\left[a_{i} c_{i}\left(c_{i}-D_{i}\right)-4 h_{i} U_{i} c_{i} r_{i}\right]}{a_{i}\left(c_{i}-D_{i}\right)^{2}} .
\end{gathered}
$$

The stability or instability discussion on this equilibrium is just like that of the third, substituting only $A_{1}$ and $B_{1}$ with $A_{2}$ and $B_{2}$.

We underline that system (6-7) is cooperative of Lotka-Volterra type, and the solution $\left(v_{i}, m_{i}\right)$ lies in the square $[0,1] \times[0,1]$. Thus the system possess one stable equilibrium at least (see [18]).

In Section 3 we will apply the model studied in this section to an environmental system situated in the Northern district of the Province of Turin (Italy). According to the analysis carried on in this section it is evident that the various scenarios admitted by the model and the asymptotic trend to a stable equilibrium solution crucially depends on the values of the model parameters. Thus, it is necessary an accurate determination of these parameters in the environment under investigation through the GIS data. This will be carried out in Section 3 where we show such an accurate determination of parameters and initial data of the state of each LU. In particular in that section we propose as well some phase diagrams (Figs. 1-6) of the system variables, where the sensitivity of the trend from the initial state to the final one can be observed.

\section{Stability analysis for each LU of the environment}

In order to check the theoretical analysis presented in the previous section, we consider the aforementioned environment of the Turin Province that has been studied in the thesis [4]. Such a system has been divided into $24 \mathrm{LUs}$, corresponding to an area of several municipalities placed around the city of Cirié.

First of all let us mention that the stability analysis carried on all the 24 LUs show that the third equilibrium, $\left(v_{i}^{(3)}, m_{i}^{(3)}\right)$, never exists since conditions (15) are not satisfied.

Moreover the analysis shows that 13 LUs have only the stable equilibrium $\left(v_{i}^{(1)}, m_{i}^{(1)}\right)$, see LUs $2,3,4$, 11, 12, 13, 14, 15, 16, 19, 21, 22, 23 in Table 1. This means that these LUs have a bad ecological state and present a strong fragmentation due to the presence of a significant edification sprawl. Therefore both bio-energy and extension of areas with a BTC index in classes $\mathcal{C}_{4}$ and $\mathcal{C}_{5}$ present a decrement of their values which asymptotically tend to zero. Such a result is somehow in accordance with the analysis carried out, with other methods, in the thesis mentioned above.

On the other hand 11 LUs exhibit stable equilibria different from $\left(v_{i}^{(1)}, m_{i}^{(1)}\right)$. For these LUs the first equilibrium is always a saddle point. In Table 1, deduced by the GIS map, we report the relevant data of the model (the area and the perimeter of the LUs are indicated in $m^{2}$ and $m$, respectively). In the last column, for each LU, we indicate which of the four equilibria results to be a stable node.

Let us note that the connectivity indexes $c_{i}$, which in the following Section 4 will be defined as functions of $M_{i}(t)$ and $M_{k}(t)$, here are assumed constant with their values recovered by the GIS data. They are computed here by formulas (26-28) of Section 4 setting $M_{i}=m_{i 0} M_{i}^{\max }$ and $M_{k}=m_{k 0} M_{k}^{\text {max }}$, for all $i, k=1, \ldots, 24$.

From the stability analysis it results that LUs $1,6,7,8,9,17,18,20$ admit the equilibrium $\left(v_{i}^{(2)}, m_{i}^{(2)}\right)$ as a saddle point and the other $\left(v_{i}^{(4)}, m_{i}^{(4)}\right)$ as a stable node. The diagrams in the phase plane show that the state of all these LUs, starting from the initial data $v_{i 0}$ and $m_{i 0}$ reported in Table 1, converges towards the fourth equilibrium which corresponds to a scenario of high ecological quality (see the examples reported below).

Conversely LUs 5 and 24 present as a unique stable node the point $\left(v_{i}^{(2)}, m_{i}^{(2)}\right)$, since $\left(v_{i}^{(4)}, m_{i}^{(4)}\right)$ does not satisfy the existence conditions of Eq.(16). Therefore the LUs show a trend towards a scenario typical of agricultural areas. 


\begin{tabular}{|c||c|c|c|c|c|c|c|c|c||c|}
\hline LU & $v_{i 0}$ & $m_{i 0}$ & $a_{i}$ & $h_{i}$ & $U_{i}$ & $c_{i}$ & $r_{i}$ & $S_{i}$ & $P_{i}$ & nodes \\
\hline \hline $\mathbf{1}$ & 0.30 & 0.19 & 1.13 & 1.83 & 0.09 & 0.12 & 0.03 & 19156648 & 25248 & $(4)$ \\
\hline $\mathbf{2}$ & 0.13 & 0.05 & 1.36 & 1.39 & 0.60 & 0.07 & 0.20 & 2260943 & 11109 & $(1)$ \\
\hline $\mathbf{3}$ & 0.20 & 0.04 & 1.43 & 0.81 & 0.41 & 0.04 & 0.14 & 764299 & 3791 & $(1)$ \\
\hline $\mathbf{4}$ & 0.25 & 0.11 & 1.27 & 1.45 & 0.25 & 0.07 & 0.08 & 2285714 & 7271 & $(1)$ \\
\hline $\mathbf{5}$ & 0.26 & 0.13 & 1.20 & 2.51 & 0.23 & 0.08 & 0.07 & 17004435 & 26335 & $(2)$ \\
\hline $\mathbf{6}$ & 0.28 & 0.27 & 1.09 & 1.36 & 0.17 & 0.20 & 0.06 & 13162369 & 22717 & $(4)$ \\
\hline $\mathbf{7}$ & 0.32 & 0.53 & 1.06 & 1.17 & 0.03 & 0.26 & 0.01 & 29896496 & 33085 & $(4)$ \\
\hline $\mathbf{8}$ & 0.26 & 0.20 & 1.12 & 1.72 & 0.23 & 0.30 & 0.08 & 10139270 & 20838 & $(4)$ \\
\hline $\mathbf{9}$ & 0.30 & 0.20 & 1.21 & 0.60 & 0.11 & 0.16 & 0.04 & 6584277 & 24162 & $(4)$ \\
\hline $\mathbf{1 0}$ & 0.21 & 0.09 & 1.43 & 1.20 & 0.36 & 0.17 & 0.12 & 837269 & 5113 & $(2),(4)$ \\
\hline $\mathbf{1 1}$ & 0.18 & 0.06 & 1.44 & 1.48 & 0.47 & 0.04 & 0.16 & 991018 & 5007 & $(1)$ \\
\hline $\mathbf{1 2}$ & 0.23 & 0.07 & 1.69 & 1.39 & 0.32 & 0.09 & 0.11 & 1395933 & 5704 & $(1)$ \\
\hline $\mathbf{1 3}$ & 0.26 & 0.10 & 1.36 & 1.81 & 0.23 & 0.07 & 0.08 & 2431435 & 7903 & $(1)$ \\
\hline $\mathbf{1 4}$ & 0.20 & 0.08 & 1.39 & 1.05 & 0.40 & 0.04 & 0.13 & 1452734 & 5483 & $(1)$ \\
\hline $\mathbf{1 5}$ & 0.11 & 0.04 & 1.62 & 1.42 & 0.66 & 0.04 & 0.22 & 1072430 & 5355 & $(1)$ \\
\hline $\mathbf{1 6}$ & 0.17 & 0.06 & 1.41 & 1.09 & 0.49 & 0.05 & 0.16 & 3416393 & 10922 & $(1)$ \\
\hline $\mathbf{1 7}$ & 0.26 & 0.13 & 1.22 & 1.58 & 0.21 & 0.14 & 0.07 & 6369795 & 12599 & $(4)$ \\
\hline $\mathbf{1 8}$ & 0.31 & 0.34 & 1.06 & 1.97 & 0.07 & 0.17 & 0.02 & 69754645 & 60482 & $(4)$ \\
\hline $\mathbf{1 9}$ & 0.13 & 0.17 & 1.24 & 1.88 & 0.61 & 0.17 & 0.20 & 4589299 & 18604 & $(1)$ \\
\hline $\mathbf{2 0}$ & 0.30 & 0.45 & 1.00 & 2.20 & 0.09 & 0.19 & 0.03 & 42953048 & 38826 & $(4)$ \\
\hline $\mathbf{2 1}$ & 0.09 & 0.02 & 2.00 & 1.06 & 0.74 & 0.06 & 0.25 & 459102 & 2887 & $(1)$ \\
\hline $\mathbf{2 2}$ & 0.05 & 0.01 & 2.00 & 1.29 & 0.86 & 0.05 & 0.29 & 302009 & 2792 & $(1)$ \\
\hline $\mathbf{2 3}$ & 0.15 & 0.05 & 1.52 & 1.27 & 0.54 & 0.05 & 0.18 & 1059134 & 4720 & $(1)$ \\
\hline $\mathbf{2 4}$ & 0.26 & 0.11 & 1.24 & 1.91 & 0.22 & 0.08 & 0.07 & 16437048 & 18458 & $(2)$ \\
\hline \hline & & & & & & & & & & \\
\hline
\end{tabular}

Table 1: Data of the environmental system and indication of the stable equilibria for each LU.

Finally LU 10 admits as stable nodes both equilibria $\left(v_{i}^{(2)}, m_{i}^{(2)}\right)$ and $\left(v_{i}^{(4)}, m_{i}^{(4)}\right)$. In this case the asymptotic behavior depends crucially on initial data. In particular with those of Table 1 the actual attractor results to be the second equilibrium, i.e. the one of agricultural scenario.

In the following figures 1-6, we show some representative behaviors through phase plane diagrams (left plots) and graphics of $v_{i}$ and $m_{i}$ versus time at an arbitrary scale (right plots). The simulations have been performed with the software Mathematica, version number 10.0.0.0.

In particular, Fig. 1 is referred to LU 5 that, as discussed previously, admits a unique stable node $\left(v_{i}^{(2)}, m_{i}^{(2)}\right)$, as it can be seen in the phase plane diagram, with $v_{i}$ tending to zero and $m_{i}$ almost constant as indicated by the time-dependent plot.

Figure 2 refers to LU 8 and shows a trend to a scenario of high ecological quality since, after a transient time, the vegetation area $v_{i}$ and the biological energy function $m_{i}$ show an increasing behavior towards the equilibrium $\left(v_{i}^{(4)}, m_{i}^{(4)}\right)$. This trend does not depend on the initial data $v_{i 0}$ and $m_{i 0}$, in the sense that $\left(v_{i}^{(4)}, m_{i}^{(4)}\right)$ represents the unique attractor of the LU, as shown in the phase diagram on the left plot, and the system asymptotically converges to such an attractor.

Figures 3 and 4 refer to LU 10 and, as indicated in Table 1, such sector shows two stable equilibria. Accordingly, the phase diagram on the left plot of Fig. 3 shows the existence of two stable nodes $\left(v_{i}^{(2)}, m_{i}^{(2)}\right)$ and $\left(v_{i}^{(4)}, m_{i}^{(4)}\right)$. The fact that the equilibrium $\left(v_{i}^{(2)}, m_{i}^{(2)}\right)$ is a stable node can be visualized with a zoom around such a point, as shown in the right frame of Fig. 3. For the initial data reported in Table 1, the left plot of Fig. 4 shows the time evolution of $v_{i}$ and $m_{i}$ towards the equilibrium $\left(v_{i}^{(2)}, m_{i}^{(2)}\right)$, which is typical of agricultural areas. The basin of attraction of the equilibrium $\left(v_{i}^{(2)}, m_{i}^{(2)}\right)$ is represented in the right plot of Fig. 4 by the grey region, showing that the initial state considered in Table 1 belongs to such basin. The case of LU 10 is very interesting from the dynamical point of view, since it represents a bistable situation. Moreover, in Section 5 when the system will be treated as a whole, the behavior of 

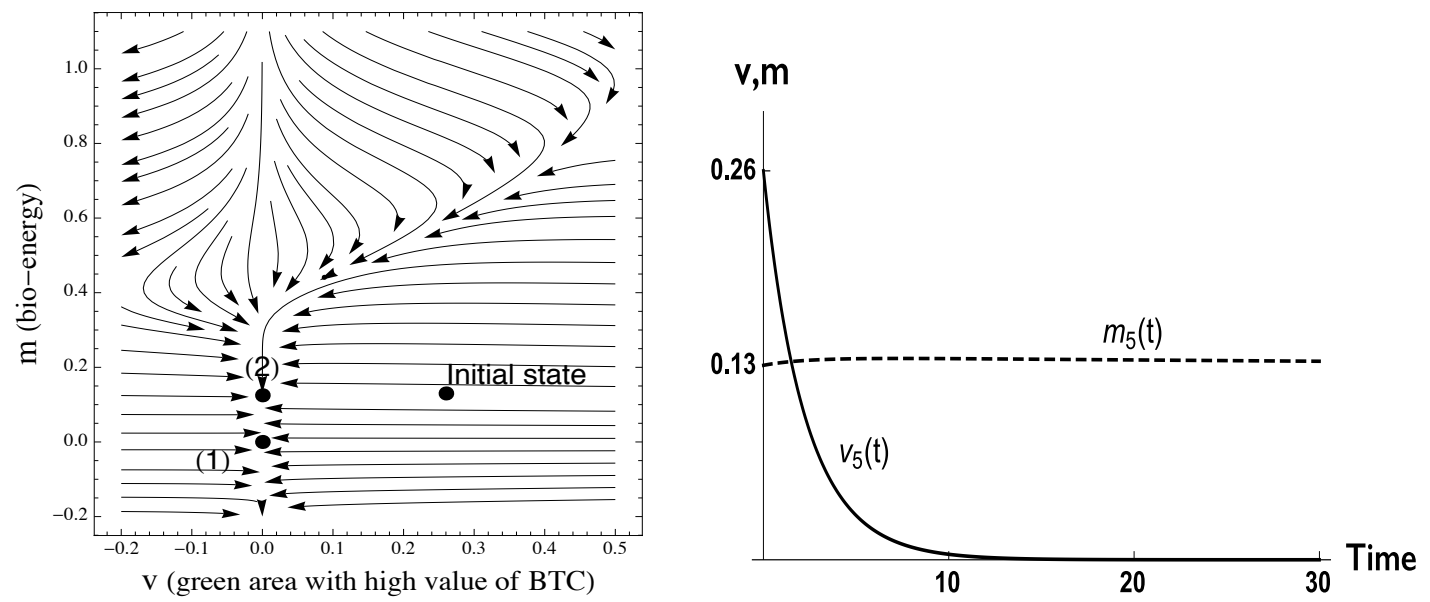

Figure 1: Representation of LU 5 (scenario typical of agricultural areas). Left - Phase diagram: state $\left(v_{5}^{(1)}, m_{5}^{(1)}\right)$ is a saddle point and state $\left(v_{5}^{(2)}, m_{5}^{(2)}\right)$ is a stable node. Right-Time evolution of $v_{5}$ and $m_{5}$ towards the equilibrium $\left(v_{5}^{(2)}, m_{5}^{(2)}\right)$.
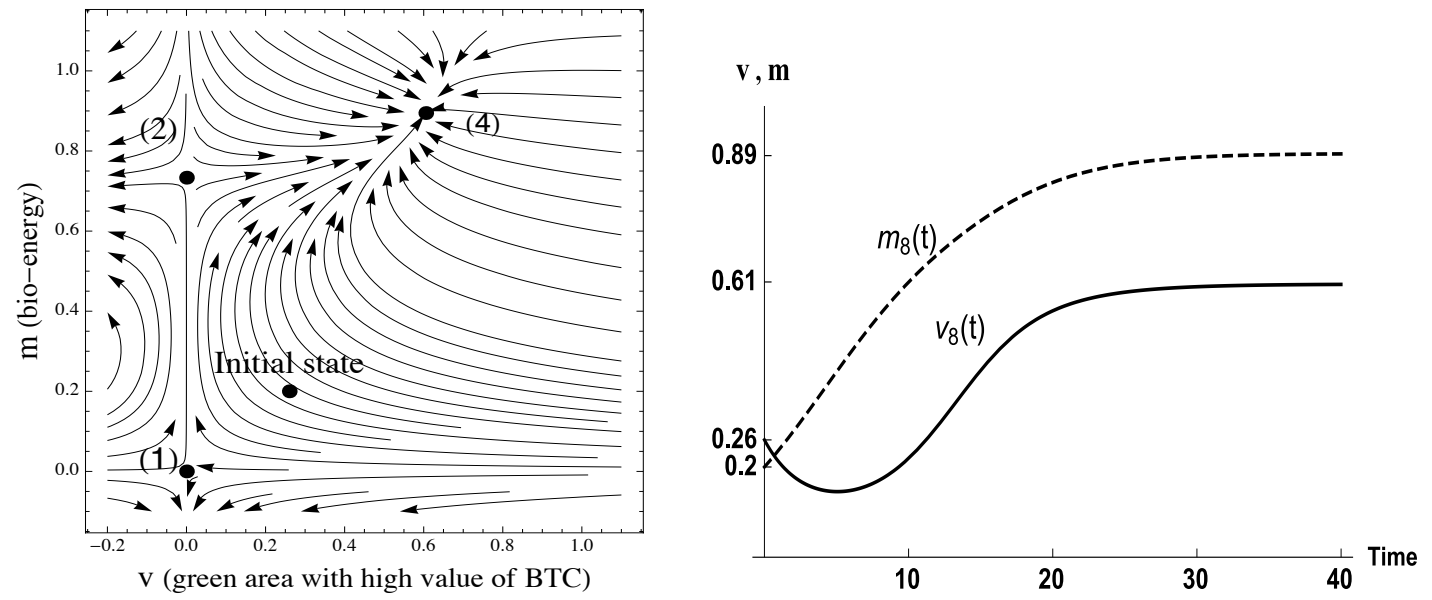

Figure 2: Representation of LU 8 (scenario of high ecological quality). Left - Phase diagram: states $\left(v_{8}^{(1)}, m_{8}^{(1)}\right)$ and $\left(v_{8}^{(2)}, m_{8}^{(2)}\right)$ are saddle points and state $\left(v_{8}^{(4)}, m_{8}^{(4)}\right)$ is a stable node. Right-Time evolution of $v_{8}$ and $m_{8}$ towards the equilibrium $\left(v_{8}^{(4)}, m_{8}^{(4)}\right)$.

LU 10 will change significantly.

Figure 5 shows the behavior of LU 13 that, as discussed before, is one of the ecological sectors presenting a strong fragmentation. Therefore the picture in the phase plane shows the existence only of the unique stable node $\left(v_{i}^{(1)}, m_{i}^{(1)}\right)$ in accordance with the time-dependent plot.

Finally, Fig. 6 corresponds to LU 20 and shows a scenario of high ecological quality, similar to the one of LU 8 represented in Fig. 2, but with a stronger trend to an equilibrium of high ecological quality. In fact, for the initial data $v_{i 0}$ and $m_{i 0}$, reported in Table 1 for this LU, the variable $v_{i}$ presents a monotonic increase from the initial state to the equilibrium $\left(v_{i}^{(4)}, m_{i}^{(4)}\right)$, conversely to that non monotonic of LU 8 .

Let us finally comment that we have also considered in our simulations the case of LU 24 which however presents exactly the same behavior as LU 5. Thus, we do not include here the plots of LU 24 . Nevertheless, when studying in Section 5 the behavior of these LUs in the whole environmental system, our numerical simulations will take into account the correct connectivity to the neighboring sectors, founding that LU 5 and LU 24 exhibit a significantly different dynamics. 

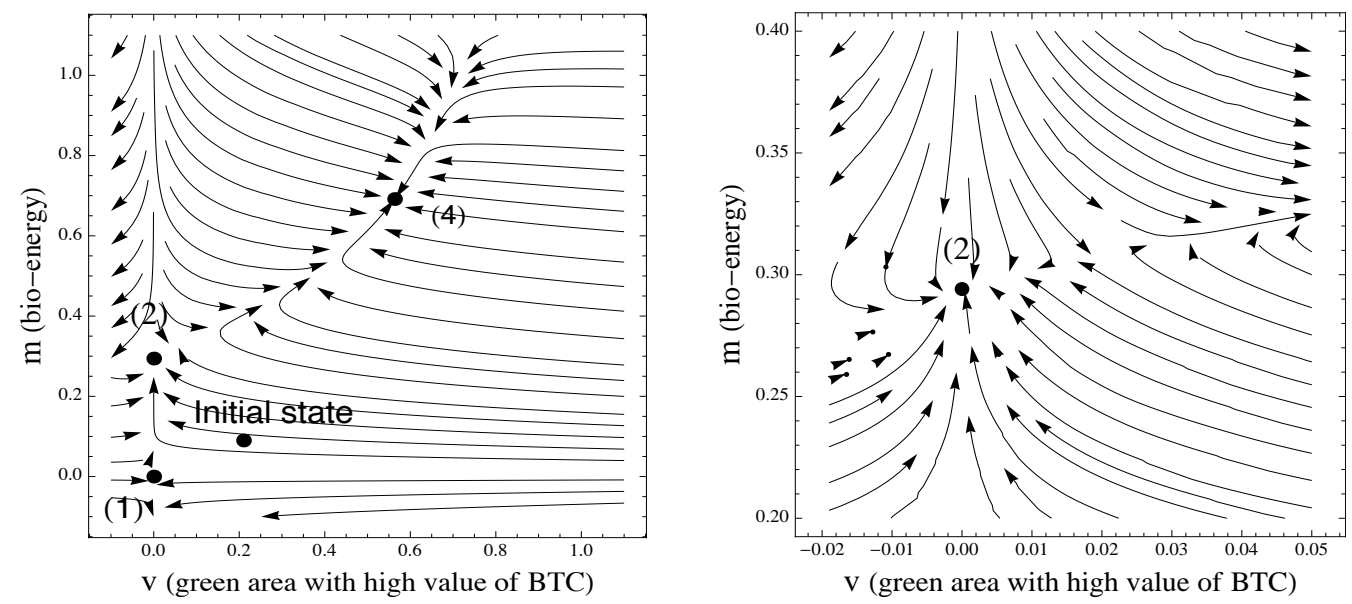

Figure 3: Representation of LU 10. Left - Phase diagram: state $\left(v_{10}^{(1)}, m_{10}^{(1)}\right)$ is a saddle point, states $\left(v_{10}^{(2)}, m_{10}^{(2)}\right)$ and $\left(v_{10}^{(4)}, m_{10}^{(4)}\right)$ are stable nodes. Right - Zoom of the phase diagram around the stable node $\left(v_{10}^{(2)}, m_{10}^{(2)}\right)$ (disregard the negative part of the picture due to the choice of the scales determined by the software Mathematica).
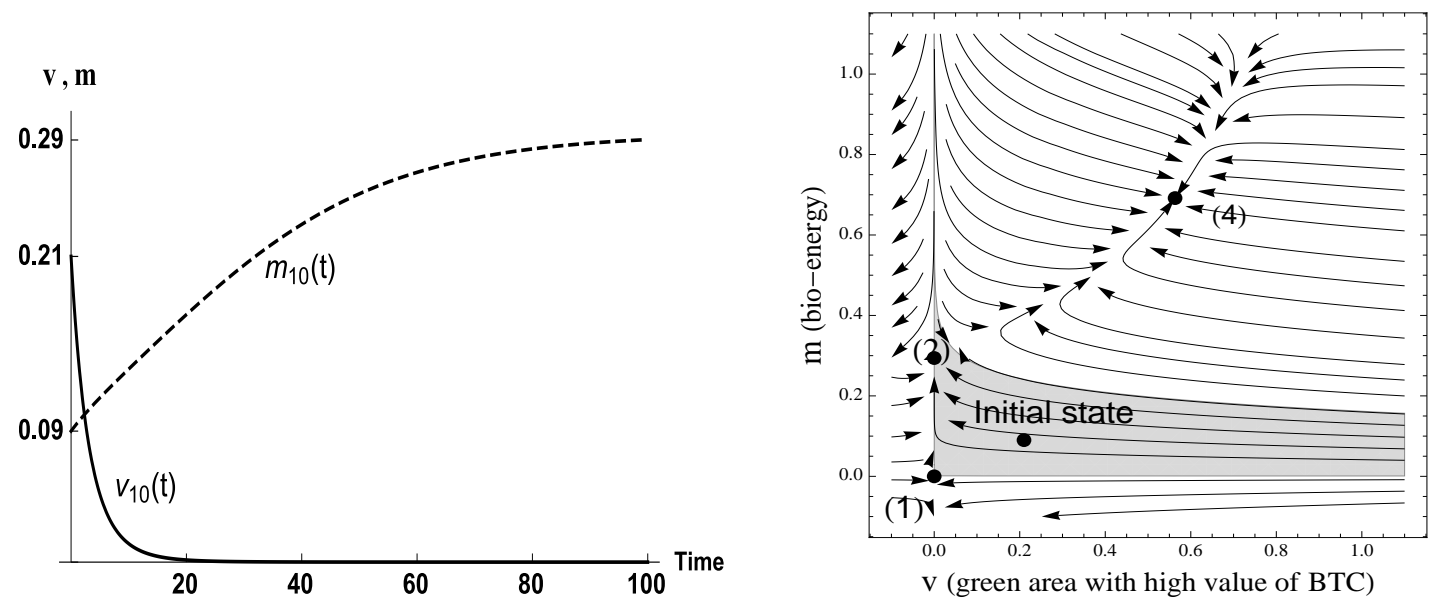

Figure 4: Representation of LU 10. Left - Time evolution of $v_{10}$ and $m_{10}$ towards the equilibrium $\left(v_{10}^{(2)}, m_{10}^{(2)}\right)$ with the initial data of Table 1. Right-Basin of attraction for the node $\left(v_{10}^{(2)}, m_{10}^{(2)}\right)$ represented by the grey region.

\section{The mathematical model extended to the whole environmen- tal system}

In this section we extend the mathematical model to the whole territory under investigation by coupling the equations of each LU with those of their neighbors. Such a coupling is determined by the connectivity indexes $c_{i}$ which can be computed through the GBTC fluxes $\Phi_{i}$ between the $i$ LUs and all their $k$ neighbors. We get [9]

$$
\Phi_{i}(t)=\sum_{k \in I_{i}} \frac{M_{i}(t)+M_{k}(t)}{2\left(P_{i}+P_{k}\right)} H_{i k}, \quad H_{i k}=\sum_{r=1}^{s} L_{i k}^{r} p^{r},
$$

where $L_{i k}^{r}$ is the length of the portion $r$ of the border with a permeability $p^{r}$. Moreover $P_{i}$ and $P_{k}$ are, as already defined, the perimeters of the two LUs and where the sum is extended to the set $I_{i}$ including 

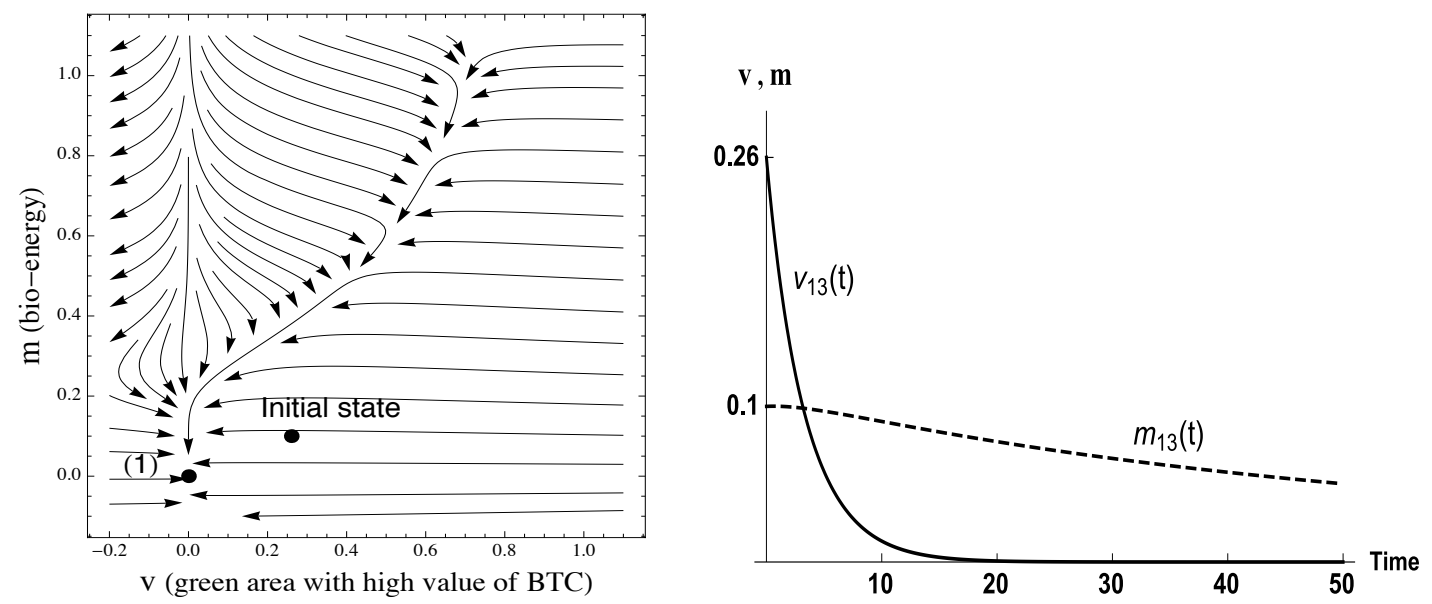

Figure 5: Representation of LU13 (scenario of ecological quality loss). Left - Phase diagram: state $\left(v_{13}^{(1)}, m_{13}^{(1)}\right)$ is the unique equilibrium and it is given by a stable node. Right-Time evolution of $v_{13}$ and $m_{13}$ towards the equilibrium $\left(v_{13}^{(1)}, m_{13}^{(1)}\right)$.
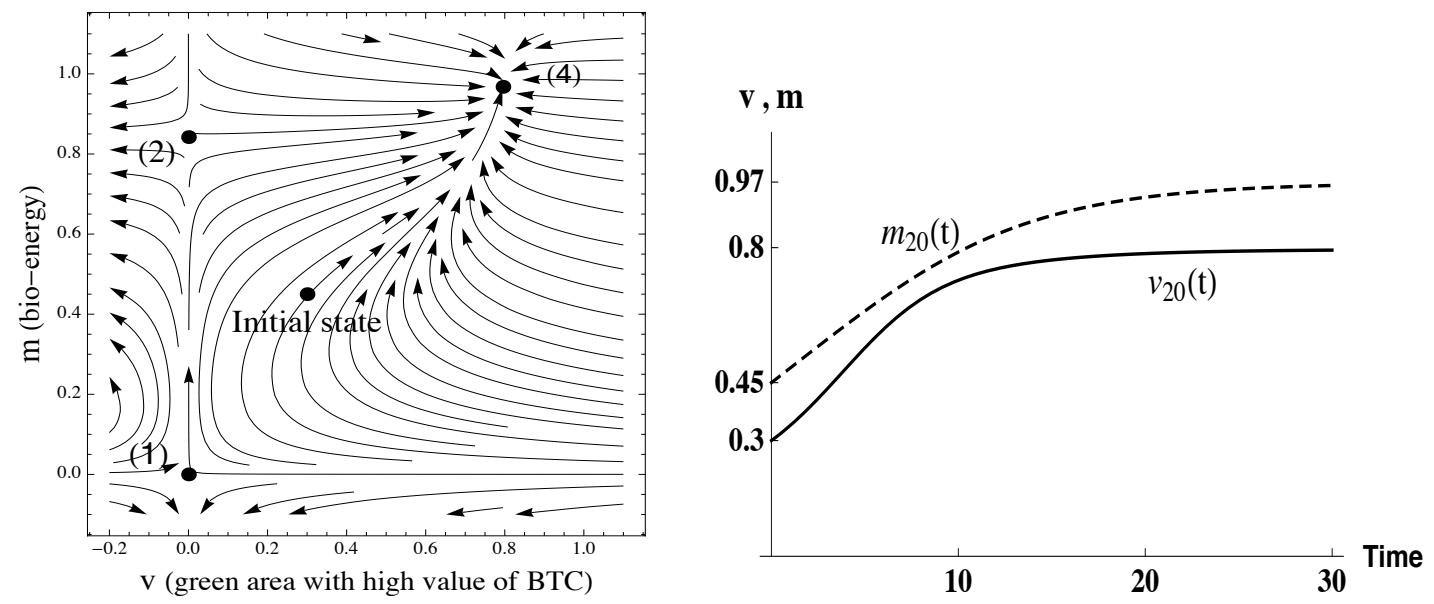

Figure 6: Representation of LU 20 (scenario of high ecological quality). Left - Phase diagram: states $\left(v_{20}^{(1)}, m_{20}^{(1)}\right)$ and $\left(v_{20}^{(2)}, m_{20}^{(2)}\right)$ are saddle points and $\left(v_{20}^{(4)}, m_{20}^{(4)}\right)$ is a stable node. Right - Time evolution of $v_{20}$ and $m_{20}$ towards the equilibrium $\left(v_{20}^{(4)}, m_{20}^{(4)}\right)$.

all the neighbors of the $i$-th LU. In addition it results

$$
\sum_{r=1}^{s} L_{i k}^{r}=L_{i k}
$$

$L_{i k}$ being the length of the border.

Taking into account that complete permeability implies $p_{r}=1$, the corresponding maximum value of $\Phi_{i}$ is given by

$$
\Phi_{i}^{\max }=\sum_{k \in I_{i}} \frac{M_{i}^{\max }+M_{k}^{\max }}{2\left(P_{i}+P_{k}\right)} L_{i k} .
$$

Finally the connectivity index of the $i$-th LU is defined by [10]

$$
c_{i}(t)=\Phi_{i}(t) / \Phi_{i}^{\max },
$$


so that $c_{i}(t) \in[0,1]$ for all $t\left(c_{i}=0\right.$ corresponds evidently to no connectivity, and $c_{i}=1$ to total connectivity).

Thus the equations of the system (6-7) are coupled through the coefficients $c_{i}(t)$ which depend on $M_{k}(t)$ and definitively on $m_{k}(t)$.

Because of the great number of LUs that an environment can possess, solving (6-7) through a numerical integrator may be costly and stiffness problems may arise. Morever in [10], the numerical integration of a system similar to that of Eqs. (6-7) has shown the presence of some instability due to the presence of such a large amount of equations. Thus, an approximation method has been there proposed in order to transform the system of ODEs in an algebraic closed hierarchy, evaluating as well its accuracy (see also [12]). The problem of solving Eqs. (6-7) through an algebraic hierarchy instead of a numerical integration allows the use of the model also by persons not acquainted with ODE integrators.

For this reason in the present paper we adopt such a method and we derive as follows such an algebraic hierarchy. If one assumes for a moment that the equations (6) and (7) are completely uncoupled, meaning that the quantities $m_{i}$ and $v_{i}$ are constant, respectively, in Eq. (6) and in Eq. (7), and that the coefficient $c_{i}$ is constant as well, then the system itself, starting from the initial data $v_{i}(t=0)=v_{i 0}$ and $m_{i}(t=0)=m_{i 0}$, has the following explicit solution, thanks to its classical logistic structure [13]

$$
\begin{aligned}
v_{i}(t) & =\frac{a_{i} m_{i}-h_{i} U_{i}}{D_{i}^{v} \exp \left[-\left(a_{i} m_{i}-h_{i} U_{i}\right) t\right]+a_{i} m_{i}} \\
m_{i}(t) & =\frac{c_{i}-r_{i}\left(1-v_{i}\right)}{D_{i}^{m} \exp \left[-\left(c_{i}-r_{i}\left(1-v_{i}\right)\right) t\right]+c_{i}}
\end{aligned}
$$

where

$$
D_{i}^{v}=\frac{a_{i} m_{i}\left(1-v_{i 0}\right)-h_{i} U_{i}}{v_{i 0}}, \quad D_{i}^{m}=\frac{c_{i}\left(1-m_{i 0}\right)-r_{i}\left(1-v_{i}\right)}{m_{i 0}} .
$$

Such a solution can be used by discretizing from $t_{0}=0$, the time axis in intervals $\Delta t=t_{s}-t_{s-1}$ sufficiently small, so that the quantities $c_{i}, v_{i}$ and $m_{i}$ can be assumed constant in the time interval $\Delta t$. Then the solution of Eqs. (6-7), by extending the formulae (29) and (30), can be approximated by the hierarchy (see the discussion in paper [10] according to the book [12])

$$
\begin{gathered}
v_{i}\left(t_{s}\right)=\frac{a_{i} m_{i}\left(t_{s-1}\right)-h_{i} U_{i}}{D_{i}^{v}\left(t_{s-1}\right) \exp \left[-\left(a_{i} m_{i}\left(t_{s-1}\right)-h_{i} U_{i}\right) \Delta t\right]+a_{i} m_{i}\left(t_{s-1}\right)} \\
m_{i}\left(t_{s}\right)=\frac{c_{i}\left(t_{s-1}\right)-r_{i}\left(1-v_{i}\left(t_{s-1}\right)\right)}{D_{i}^{m}\left(t_{s-1}\right) \exp \left[-\left(c_{i}\left(t_{s-1}\right)-r_{i}\left(1-v_{i}\left(t_{s-1}\right)\right)\right) \Delta t\right]+c_{i}\left(t_{s-1}\right)}
\end{gathered}
$$

where

$$
D_{i}^{v}\left(t_{s-1}\right)=\frac{a_{i} m_{i}\left(t_{s-1}\right)\left[1-v_{i}\left(t_{s-1}\right)\right]-h_{i} U_{i}}{v_{i}\left(t_{s-1}\right)}, \quad D_{i}^{m}\left(t_{s-1}\right)=\frac{c_{i}\left(t_{s-1}\right)\left[1-m_{i}\left(t_{s-1}\right)\right]-r_{i}\left[1-v_{i}\left(t_{s-1}\right)\right]}{m_{i}\left(t_{s-1}\right)} .
$$

Of course the determination of such a hierarchy at time $t_{s}$ must take into account that for any solution $m_{i}$ at time $t_{s-1}$ it is necessary to compute, for all the LUs, the values of the GBTC $M_{i}=m_{i} M_{i}^{\max }$, of the fluxes $\Phi_{i}$ and then of the connectivity indexes $c_{i}$. On the other hand the values of $h_{i}, U_{i}, r_{i}, M_{i}^{\max }$ and $H_{i k}$ are constant and consequently can be computed before the generation of the hierarchy itself.

Finally, it is worthwhile to note that the hypothesis that territorial quantities are almost constant during the time interval $\Delta t$ is justified by the fact the relaxation time of an environmental system is sufficiently long. 


\section{Evaluation analysis of the whole environmental system}

In this section we examine the dynamics of the whole environmental system, providing the solution obtained with the iterative scheme explained in the previous section, using Eqs. (31-32). The computations have been performed using the software Mathematica, version number 10.0.0.0. We give in the Table 2 the values of the quantities $H_{i k}$ and $L_{i k}$. Note that in the first column of the table the couple of the neighboring LUs is indicated only for those LUs that present permeable borders.

\begin{tabular}{|l||r|r|}
\hline LUs & $H_{i k}$ & $L_{i k}$ \\
\hline \hline $1 \_2$ & 391 & 782 \\
\hline $1 \_17$ & 3017 & 7543 \\
\hline $1 \_18$ & 3664 & 7327 \\
\hline $1 \_24$ & 2896 & 5792 \\
\hline $2 \_3$ & 1098 & 1569 \\
\hline $2 \_4$ & 1292 & 1845 \\
\hline $4 \_5$ & 964 & 1377 \\
\hline $4 \_15$ & 1062 & 2654 \\
\hline $5 \_2$ & 262 & 374 \\
\hline $5 \_6$ & 3837 & 7675 \\
\hline $5 \_9$ & 2804 & 7011 \\
\hline \hline
\end{tabular}

\begin{tabular}{|l||r|r|}
\hline LUs & $H_{i k}$ & $L_{i k}$ \\
\hline \hline $5 \_14$ & 553 & 1383 \\
\hline $5 \_15$ & 633 & 1583 \\
\hline $5 \_21$ & 267 & 381 \\
\hline $5 \_22$ & 122 & 175 \\
\hline $6 \_7$ & 5416 & 9027 \\
\hline $6 \_8$ & 533 & 927 \\
\hline $7 \_8$ & 1460 & 2919 \\
\hline $8 \_9$ & 4231 & 8991 \\
\hline $9 \_11$ & 241 & 602 \\
\hline $9 \_12$ & 1931 & 2758 \\
\hline $9 \_13$ & 1072 & 1531 \\
\hline
\end{tabular}

\begin{tabular}{|l||l|r|}
\hline LUs & $H_{i k}$ & $L_{i k}$ \\
\hline \hline $9 \_23$ & 771 & 1543 \\
\hline $10 \_9$ & 425 & 850 \\
\hline $10 \_20$ & 305 & 763 \\
\hline $11 \_12$ & 855 & 2137 \\
\hline $11 \_19$ & 228 & 1138 \\
\hline $12 \_13$ & 984 & 2461 \\
\hline $13 \_19$ & 821 & 1172 \\
\hline $13 \_23$ & 680 & 3401 \\
\hline $14 \_15$ & 634 & 1585 \\
\hline $14 \_16$ & 548 & 1096 \\
\hline $14 \_22$ & 109 & 156 \\
\hline \hline
\end{tabular}

\begin{tabular}{|l||r|r|}
\hline LUs & $H_{i k}$ & $L_{i k}$ \\
\hline \hline $16 \_17$ & 1785 & 3570 \\
\hline $16 \_21$ & 311 & 778 \\
\hline $16 \_22$ & 281 & 702 \\
\hline $18 \_17$ & 5429 & 10857 \\
\hline $18 \_19$ & 3352 & 6705 \\
\hline $18 \_20$ & 5390 & 10780 \\
\hline $19 \_20$ & 1553 & 3106 \\
\hline $21 \_22$ & 184 & 459 \\
\hline $21 \_23$ & 197 & 493 \\
\hline $24 \_2$ & 2557 & 5114 \\
\hline & & \\
\hline
\end{tabular}

Table 2: Values of $H_{i k}$ and $L_{i k}$ for the neighboring LUs presenting permeable borders.

Figures 7,8 and 9 show the time evolution of $v_{i}$ and $m_{i}$ (for $i=5,8,10,13,20,24$ ), at an arbitrary scale, when the corresponding LUs are connected in the whole environmental system, through time dependent connectivity indexes $c_{i}(t)$.
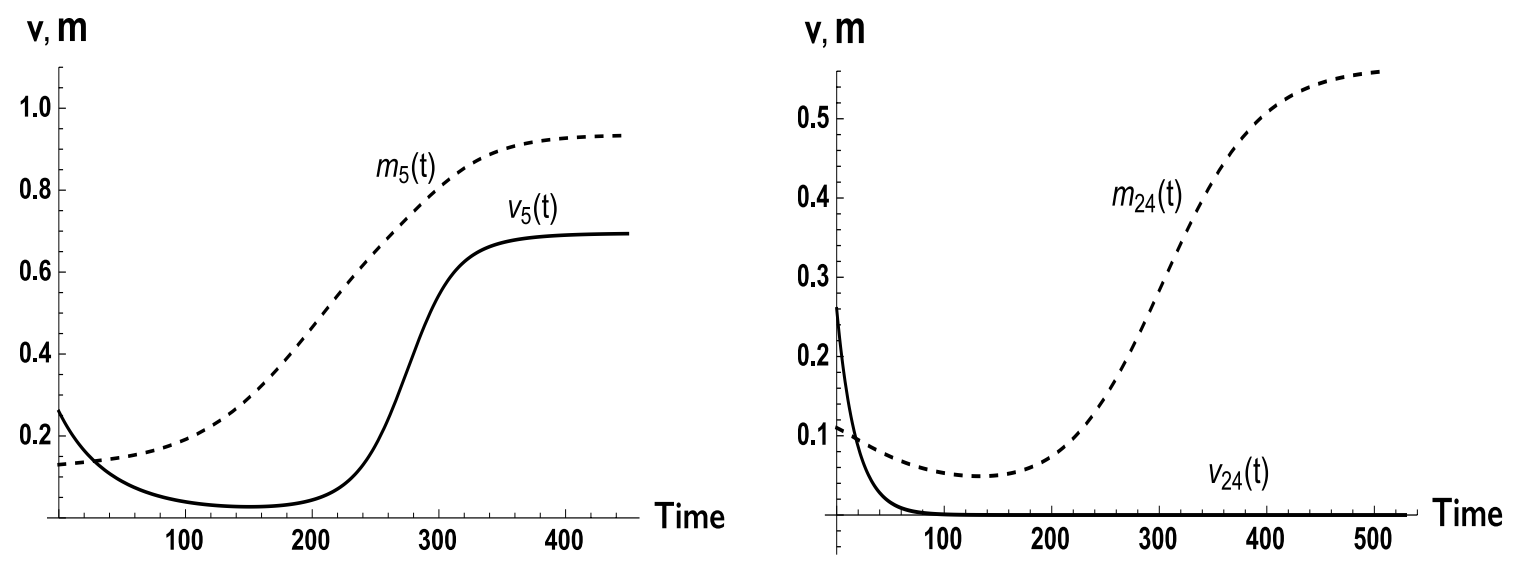

Figure 7: Representation of the LUs in the whole environmental system. Left - LU 5. Evolution of $v_{5}$ and $m_{5}$ versus time. Right - LU 24. Evolution of $v_{24}$ and $m_{24}$ versus time.

In particular Fig. 7 presents the time evolution of LU 5 and LU 24. In Section 3, when studying separately the landscape units, we have mentioned that these two LUs exhibit the unique stable node $\left(v_{i}^{(2)}, m_{i}^{(2)}\right)$, see Table 1, and have a similar behavior, so that we have shown only the plots of Fig. 1 concerning LU 5. The behavior of these LUs is completely different when the environmental system is considered as a whole. In fact, a new stable node of high ecological quality appears for LU 5 , say $\left(v_{5}^{(4)}, m_{5}^{(4)}\right)$, thanks to the monotonic increasing of the GBTC variable $m_{5}$. Despite the fact that for a long initial transient the variable $v_{5}$ assumes values close to zero, LU 5 tends to the node $\left(v_{5}^{(4)}, m_{5}^{(4)}\right)$. Conversely LU 24 , because of a significant decreasing of $m_{24}$ during the transient behavior, does not reach an equilibrium of high ecological quality and leads to the one typical of agricultural areas. 

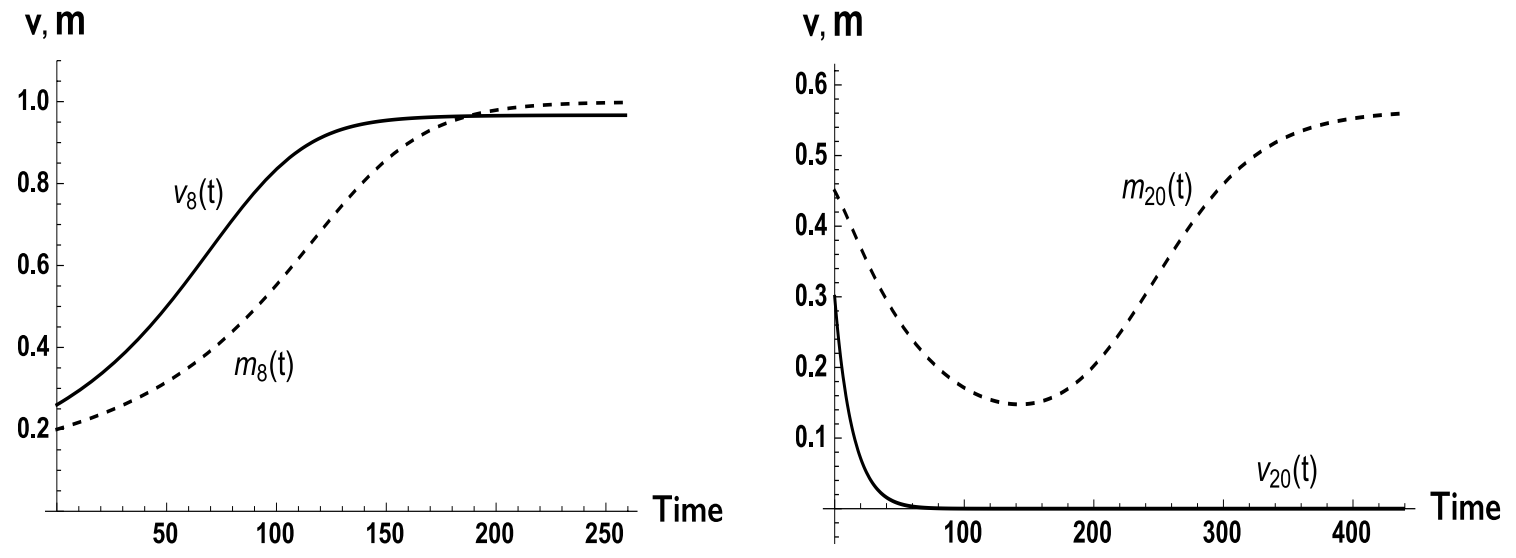

Figure 8: Representation of the LUs in the whole environmental system. Left-LU 8. Evolution of $v_{8}$ and $m_{8}$ versus time. Right - LU 20. Evolution of $v_{20}$ and $m_{20}$ versus time.

Moreover Fig. 8 describes the dynamics of LU 8 and LU 20 which, when isolated, exhibit a trend to the stable nodes $\left(v_{i}^{(4)}, m_{i}^{(4)}\right)$ of high ecological quality, as shown in Figures 2 and 6 of Section 3. In the whole system simulation, LU 8 still reaches the equilibrium $\left(v_{8}^{(4)}, m_{8}^{(4)}\right)$, whereas LU 20 presents a loss of ecological quality and evolves towards the equilibrium $\left(v_{20}^{(2)}, m_{20}^{(2)}\right)$, since for a long time interval it exhibits a strong decrease of the GBTC variable $m_{20}$ which causes the decay to zero of the variable $v_{20}$.
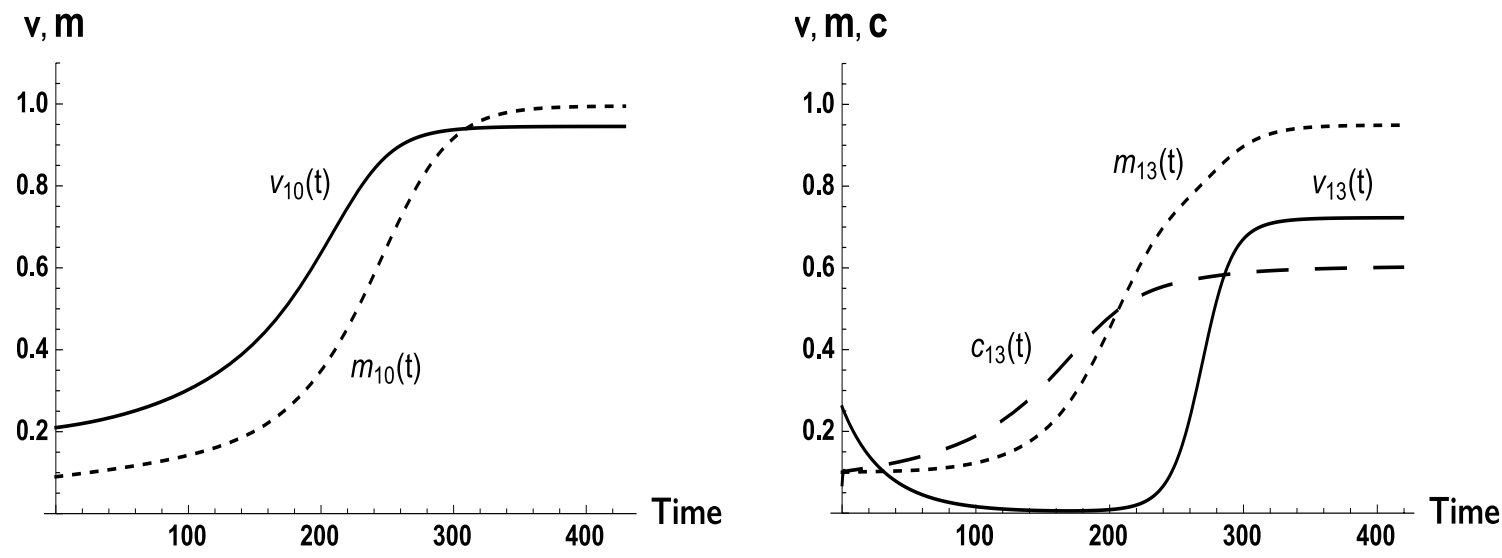

Figure 9: Representation of the LUs in the whole environmental system. Left-LU 10. Evolution of $v_{10}$ and $m_{10}$ versus time. Right - LU 13. Evolution of $v_{13}, m_{13}$ and $c_{13}$ versus time.

Finally, Fig. 9 considers LU 10 and LU 13. The stability analysis carried out in Section 3 for LU 10 shows, as visualized by Fig. $3-$ left, that this sector admits the two stable attractors $\left(v_{10}^{(2)}, m_{10}^{(2)}\right)$ and $\left(v_{10}^{(4)}, m_{10}^{(4)}\right)$, and consequently the dynamics of such LU depends strongly on the initial data (in particular for those of Table 1 the equilibrium solution reached was $\left.\left(v_{10}^{(2)}, m_{10}^{(2)}\right)\right)$. When the whole system is considered, LU 10 improves its environmental properties and tends to a scenario of high ecological quality reaching the equilibrium $\left(v_{10}^{(4)}, m_{10}^{(4)}\right)$, since evidently the basin of attraction of node $\left(v_{10}^{(2)}, m_{10}^{(2)}\right)$ is now different. Analogously, also LU 13 presents such an improvement when well connected to its neighbors, as it can be seen by Fig. 9-right: after a long initial transient where the variable $v_{13}$ is close to zero, then there is a strong growth of $v_{13}$ itself due to the monotonic increasing of both the GBTC variable $m_{13}$ and the connectivity index $c_{13}$ (dashed line). Thus, LU 13 changes from a scenario showing a complete loss of ecological quality, defined by the stable node $\left(v_{13}^{(1)}, m_{13}^{(1)}\right)$ when it is isolated, to an opposite scenario of 
high ecological quality stated by $\left(v_{13}^{(4)}, m_{13}^{(4)}\right)$, when it is considered connected to the whole environmental system. Such examples evidence how a LU can commute to a scenario of high ecological quality when it is well connected to its neighbors, even when a bad trend is found in the stability analysis developed individually for each LU. Such a behavior influences also the time scaling of the system. Let us note in fact that the time scales of figures 7,8 and 9 are much greater than those provided when the LUs were considered separately. This peculiarity seems reasonable since, when the environmental system is treated as a whole, then the relaxation times increase and fluctuations of territorial quantities get slower.

\section{Conclusions and future perspectives}

In this paper we have proposed a new mathematical model for the evaluation of the ecological state of an environmental system distributed in landscape units (LUs). More specifically, starting from previous ideas advanced in paper [9], our model acts at the level of each LU, instead at that of the whole system, and introduces as state variables the extent of green area of high ecological quality and a generalized biological energy of each landscape unit. The model is then capable to describe the territory at a more detailed level, so that its properties are better apprehended. From the mathematical point of view, the model is represented by an autonomous system of ordinary differential equations of cooperative LotkaVolterra type [14]. The stability analysis developed in Section 2 for each LU determines the equilibrium solutions of the equations, whose qualitative trend indicates the future possible scenarios of the LU itself. The analysis is then applied in Section 3 to a Northern district of the Turin Province consisting of 24 different ecological sectors linked through a constant connectivity index recovered from the GIS. It allows to identify the LUs with high ecological quality, showing a great potential to evolve to a favorable scenario, and, conversely, those presenting a bad ecological state with a tendency to a scenario of ecological quality loss. It also allows to identify the LUs showing a different asymptotic equilibrium, in particular that typical of agricultural areas.

For a comprehensive description of the whole environmental system, the dynamics of the landscape units is then investigated in Section 4, considering all LUs connected to their neighboring sectors. The resulting model incorporates the connectivity issues among the neighboring sectors showing that the connectivity index plays an important role since now it has become time dependent through the state variables. The analytical treatment of such a system is rather complicated, since it is represented by 48 coupled ordinary differential equations. Our strategy was then to use an approximation procedure based on an algebraic hierarchy and, following the ideas discussed in paper [10], we propose an explicit algorithm presented in Section 5. The simulations show how a LU can commute from a certain scenario to a completely different situation, due to the influence of its neighbors, even if a different trend is predicted in the stability analysis developed individually for each LU.

Therefore, the study developed in this paper indicates that the connectivity among the neighboring sectors has a significant impact in the dynamics of the LUs when they are considered as parts of a whole. Even a rather complete analysis of the individual LUs is not enough to describe the whole system and a model taking into account the connectivity issues is an appropriate tool.

In our opinion, the model proposed here offers promising results and motivates future perspectives in terms of networking systems accounting for neighboring sectors. In fact, we think that it is possible to propose a model similar to the one presented here for what concerns the state variables, but different for the LUs coupling, borrowing some ideas from electrical synapses linking neurons [2] and exploiting the analysis of the landscape connectivity [6]. Moreover, another development could take into account a model with more state variables, namely considering variables $v$ 's for each ecological class $\mathcal{C}_{2}, \ldots, \mathcal{C}_{5}$ with non-null BTC indexes. In such a way, the model would also include the effects due to the presence of landcover areas with weak production of biological energy. These developments can be introduced in a forthcoming paper.

Acknowledgments. The present research has been partially supported by the National Group GNFM of INdAM. One of the Authors (AJS) thanks this institution for the financial support given in his visiting professor program in Italy. 


\section{References}

[1] Argent R.M., Sojda R.S., Guipponi C., McIntosh B., Voinov A.A, Maier H. R. (2016), Best practices for conceptual modelling in environmental planning and management. Environmental Modelling \& Software, 80: 113-121.

[2] Boccaletti S., Latora V., Moreno Y., Chavez M., Hwang D. U. (2006), Complex networks: Structure and dynamics. Physics Reports, 424: 175-308.

[3] Cassatella C, Peano A. (eds) (2011), Landscape Indicators: Assessing and Monitoring Landscape Equality. Dordrecht: Springer.

[4] De Palma I. (2014), Methodologies of Landscape Ecology and Mathematical Models for Environments Evaluation and Ecoservice Systems Estimate, Master Degree Thesis, Politecnico di Torino.

[5] Fabbri P. (2007), Principi Ecologici per la Progettazione del Paesaggio (Ecological Principles of Landscape Planning). Milano: Franco Angeli.

[6] Foltête J.-C., Clauzel C., Vuidel G. (2012), A software tool dedicated to the modelling of landscape networks. Environmental Modelling \& Software, 38: 316-327.

[7] National Geographic website (visited in November 2016), http://nationalgeographic.org/ encyclopedia/geographic-information-system-gis/

[8] Gobattoni F., Lauro G., Leone A., Monaco R., Pelorosso R. (2011), A procedure for the mathematical analysis of landscape evolution and scenarios assessment. Landscape \& Urban Planning, 103: 289302 .

[9] Gobattoni F., Lauro G., Monaco R., Pelorosso R. (2013), Mathematical models in landscape ecology: stability analysis and numerical tests. Acta Appl. Math., 125: 173-192.

[10] Gobattoni F., Groppi M., Monaco R., Pelorosso R. (2014), New developments and results for mathematical models in environment evaluations. Acta Appl. Math., 132: 321-331.

[11] Ingegnoli V. (2002), Landscape Ecology: a widening foundation. Berlin: Springer-Verlag.

[12] Ixaru L.G. (1984), Numerical Methods for Differential Equations and Applications. Dordrecht: Reidel.

[13] Jordan, D.W., Smith, P.(1977), Nonlinear Ordinary Differential Equations. Oxford: Clarendon Press.

[14] Murray J.D. (2002) Mathematical Biology. I. An Introduction. Berlin: Springer-Verlag.

[15] O’Neill R.V.et al. (1988), Indices of landscape pattern. Landscape Ecology, 3:153-162.

[16] Pielou E.C. (1975), Ecological diversity, New York: John Wiley.

[17] Shannon C.E., Weaver W. (1949), The Mathematical Theory of Communication. Urbana: University of Illinois Press.

[18] Smith H.L. (2008), Monotone dynamical systems: an introduction to the theory of competitive and cooperative systems. No.41. American Mathematical Society.

[19] Turner M.G. (1989), Landscape Ecology: the effect of pattern on process. Annual Review of Ecology and Systematics, 20: 171-197. 